\title{
Contingency Management Approaches for Adolescent Substance Use Disorders
}

\author{
Catherine Stanger, PhD [Associate Professor] ${ }^{\mathrm{a}}$ and Alan J. Budney, PhD [Professor] ${ }^{\mathrm{b}}$ \\ aDepartment of Psychiatry, University of Arkansas for Medical Sciences, Little Rock, Arkansas \\ ${ }^{b}$ Department of Psychiatry, University of Arkansas for Medical Sciences, Little Rock, Arkansas
}

\section{Abstract}

The addition of contingency management (CM) to the menu of effective treatments for adolescent substance abuse has generated excitement in the research and treatment communities. CM interventions are based on extensive basic science and clinical research evidence demonstrating that drug use is sensitive to systematically applied consequences. This article provides (a) a review of basic CM principles, (b) implementation guidelines, (c) a review of the clinical CM research targeting adolescent substance abuse, and (d) a discussion of implementation successes and challenges. Although the research base for $\mathrm{CM}$ with adolescents is in its infancy, there are multiple reasons for high expectations.

\section{Keywords}

contingency management; adolescent substance abuse; marijuana; parenting

\begin{abstract}
An emerging literature indicates that adolescents in treatment for substance abuse have better outcomes than those not in treatment, and suggests that multiple types of behavioral interventions hold promise ${ }^{1}$. Treatments with empirical support from well-designed, randomized clinical trials include: group and individual cognitive-behavioral therapy (CBT), CBT combined with motivational enhancement therapy (MET), community reinforcement approach counseling (CRA), Functional Family Therapy, Multidimensional Family Therapy (MDFT), Multisystemic Therapy (MST), Brief Strategic Family Therapy (BSFT); Family Support Network (FSN), and Family Behavior Therapy. The MET and CBT interventions that have been tested are similar in scope and duration to those used with adults. The familyfocused interventions take advantage of social networks (parents, schools, and other social agencies) that are somewhat unique to adolescents. These generally include efforts to address and alter maladaptive family patterns that contribute to substance use (e.g. parent drug use, parent-child relationships, parent supervision), make use of resources in the school and criminal justice system, and address problems that might be associated with the child's peer network. Although yet to be clearly supported by the empirical literature ${ }^{2,3}$, some
\end{abstract}

\footnotetext{
Corresponding author for proof and reprints: Catherine Stanger, PhD, Department of Psychiatry, University of Arkansas for Medical Sciences, 4301 West Markham St. \#843, Little Rock, AR 72205, (501) 526-8444, (501) 526-7816 (fax), cstanger@uams.edu. Coauthor address: Alan Budney, PhD, Department of Psychiatry, University of Arkansas for Medical Sciences, 4301 West Markham St. \#843, Little Rock, AR 72205, (501) 526-8443, (501) 526-7816 (fax), ajbudney@uams.edu

Publisher's Disclaimer: This is a PDF file of an unedited manuscript that has been accepted for publication. As a service to our customers we are providing this early version of the manuscript. The manuscript will undergo copyediting, typesetting, and review of the resulting proof before it is published in its final citable form. Please note that during the production process errors may be discovered which could affect the content, and all legal disclaimers that apply to the journal pertain.
} 
assert that these behaviorally-based, family approaches produce a more potent effect than those that do not include a family component.

That said, even with the most potent interventions tested to date, reductions in substance use observed have been modest, and robust effects on abstinence rates have been difficult to demonstrate. For example, the largest published clinical trial included adolescents aged 12 to 18 who endorsed at least 1 criterion for cannabis abuse or dependence and had used cannabis in the previous 90 days $^{2}$. Adolescents received one of five treatment treatments across four clinical sites: MET/CBT5 (2 individual and 3 group sessions), MET/CBT12 (2 individual and 10 group sessions), MET/CBT12 plus Family Support Network, Adolescent CRA, and MDFT. Significant decreases in drug use and symptoms of dependence were observed following each of the treatments. However, robust between-treatment differences in outcomes were not observed. Although reductions in drug use were promising compared with that observed in prior treatment studies, approximately two-thirds of the youth continued to experience significant substance-related symptoms. That is, many never achieve abstinence or substantial reductions in cannabis use, and many of those who are initially successful relapse. These findings, combined with the similarly modest effects demonstrated in studies examining the family-focused and individual/group therapies mentioned above, indicate a strong need for continued exploration and development of more potent adolescent treatment models and interventions ${ }^{4}$. Moreover, few studies have attempted to isolate active components of multi-faceted treatments and little is know about mechanisms of action for the positive effects observed across studies ${ }^{1}$.

One alternative candidate for enhancing outcomes with a strong probability of success is contingency-management based interventions (CM). CM interventions are based on extensive basic science and clinical research evidence demonstrating that drug use and abuse are sensitive to systematically applied environmental consequences, i.e., reinforcement and punishment contingencies ${ }^{5}$. CM approaches have become one of the most thoroughly researched and effective behavioral procedures to increase drug abstinence and other treatment targets across adult substance-dependence disorders ${ }^{6-8}$; however, such interventions, as of yet, received only minimal attention in the adolescent treatment literature. Clinical studies across multiple, adult substance dependent populations have demonstrated the efficacy of various types of CM interventions for increasing (1) abstinence from alcohol, cannabis, cocaine, methamphetamine, and opiates; (2) counseling attendance and retention in treatment; (3) compliance with medications like naltrexone; and (4) completion of therapeutic activities.

\section{Contingency Management Conceptualization and Basic Principles}

$\mathrm{CM}$ applications are derived from an operant framework, in which substance use is considered a case of operant behavior that is maintained, in part, by the pharmacological actions of the substance in conjunction with social and other non-pharmacological reinforcement derived from a substance using lifestyle. An important feature of this conceptual model is that it facilitates a direct connection between clinical practice and the scientific disciplines of behavior analysis and behavioral pharmacology. Those disciplines include an extensive research literature demonstrating principles and procedures that can be applied to modify behavior of all kinds, including substance use and misuse. A major strength of conceptualizing substance abuse as an operant behavior is that, as such, it is amenable to change via the same processes and principles as other types of human behavior irrespective of its etiology. The treatment goal is to systematically weaken the influence of reinforcement derived from substance use and its related lifestyle, and to increase the frequency and magnitude of reinforcement derived from healthier alternative activities, especially those that are incompatible with continued substance use. 
The CM approach capitalizes on knowledge that drug-seeking and drug-use can be directly modified by manipulating the relevant environmental contingencies. Typically, $\mathrm{CM}$ interventions are used to engender therapeutic change within a comprehensive treatment program in a substance abuse treatment clinic. CM programs arrange the therapeutic environment such that (a) target therapeutic behaviors such as drug abstinence, counseling attendance, and medication compliance are carefully monitored, and (b) reinforcing or punishing events (e.g., tangible rewards or incentives, suspension of employment or school, loss of privileges) occur when the target behavior is or is not achieved.

Within the adult substance abuse treatment literature, $\mathrm{CM}$ interventions have primarily involved the use of positive reinforcement, however, negative reinforcement, positive punishment, and negative punishment contingencies can also motivate increases and decreases in therapeutic target goals. Here we provide clarification of these 4 basic processes that can be used to design effective CM interventions. Positive reinforcement involves delivery of a desired consequence (e.g., tangible goods or services, social praise, increased privileges) contingent on meeting a therapeutic goal (e.g., negative urinalysis test results, attendance at a counseling session). Negative reinforcement involves removing an aversive or confining circumstance (e.g., criminal justice supervision, early curfew, grounding) contingent on meeting a therapeutic goal. Positive punishment involves delivery of a punishing consequence (e.g., suspension for school, loss of phone or computer use, grounding) contingent on evidence of undesirable behavior (e.g., positive urinalysis test result). Negative punishment involves removal of a positive circumstance or condition (e.g., reduction in the value of rewards that could be earned, removal or reduction of privileges) contingent on evidence of the occurrence of an undesirable behavior.

Both reinforcement and punishment contingencies can be effective tools in substance abuse treatment programs. Typically use of positive reinforcement is preferred over punishment. Caution is indicated when considering use of punishment contingencies without careful planning, as these may inadvertently increase undesirable behaviors such as treatment dropout or negatively impact therapeutic relationships. Almost all CM applications developed for adults have utilized positive reinforcement principles ${ }^{5}$.

\section{Contingency Management Implementation}

Behavioral analytic theory and the empirical literature on behavior change suggests that the efficacy of CM interventions will be influenced by 5 variables: the schedule used to deliver consequences, the magnitude of the consequence, the choice of the target behavior, the selection of the type of consequence, and the monitoring of the target behavior ${ }^{9}$ (see Figure 1). The schedule of reinforcement or punishment refers to the temporal relation between the target behavior and the delivery of the consequence. Generally, efficacy is likely to improve as the temporal delay between the occurrence of the target behavior and delivery of the consequence decreases. For example, all else being equal, providing positive reinforcement for drug abstinence five minutes after a client submits a negative urine specimen would likely engender greater rates of abstinence than waiting a week before reinforcement is delivered. Similarly, more frequent schedules of reinforcement are usually preferable to less frequent schedules in establishing an initial target behavior like drug abstinence or regular attendance at counseling sessions. Frequent schedules allow multiple opportunities to reinforce and thereby strengthen the target behavior. Once a target behavior is established, less frequent schedules are typically considered for maintenance of behavior change. Two schedules that have demonstrated efficacy across multiple substance abuse treatment studies are a fixed schedule with escalating rewards and a reset contingency (typically referred to as abstinence-based vouchers or incentives ${ }^{5}$ ) and an intermittent schedule of rewards using the 
fishbowl method ${ }^{10}$. Both schedules are described in more detail below in the context of our adolescent CM program.

The magnitude of reinforcement or punishment is also an important factor that can affect the efficacy of CM interventions. For example, if the goal is drug abstinence, a $\$ 10$ incentive for each negative drug test is likely to be more effective in increasing abstinence than one worth $\$ 2.00$. Given the resilience of substance use, strong reinforcers may be necessary to compete with the reinforcement derived from well-established use patterns. Multiple studies have demonstrated that greater magnitude schedules of reinforcement have resulted in better abstinence outcomes than lower magnitude ${ }^{11}$. Creative use of relatively low magnitude reinforcers and variable or intermittent schedules can successfully modify target behaviors among drug abusers ${ }^{12}$. However, as one would expect, the larger the incentives the higher probability of motivating behavior change in a greater proportion of cases. In addition, larger magnitude incentives have been shown to be more cost effective than lower magnitude incentives ${ }^{13}, 14$.

The type of reinforcers or punishers used in a CM program can be critical to its success. Individuals vary greatly in terms of the types of goods and services that will serve as reinforcers. For example, a specific reinforcer (e.g., pizza or movie theatre passes) that serves as an effective incentive for one client may not be reinforcing for another. Use of a range of incentives or allowing clients to choose their incentive can increase the probability that the incentive will serve as a reinforcer and facilitate the desired target behavior. Incentives used in adult CM programs have ranged from cash, choice of gift cards, on-site retail items, increase chance to receive prizes, desirable clinic privileges, employment or housing opportunities, and refunds on treatment service fees ${ }^{8}$. Of course each of these has its strengths and drawbacks, and must be tailored to the clinic's needs and limitations.

The most commonly selected target behavior used in adult CM programs has been drug abstinence. CM programs, however, have also targeted medication compliance, counseling attendance, and completion of lifestyle change activities. When choosing targets, one should be aware that successful change in one behavior may not result in change in another. For example, treatment attendance may improve by providing incentives for coming to sessions, but drug use might not be affected ${ }^{15}$. The extant adult CM literature suggests that the first choice of a target behavior should be drug use. When selecting other targets, these should be specific, individualized behavioral goals that have a high probability for successful completion.

Effective monitoring of the targeted behavior is essential to a CM program, because consequences (reinforcement or punishment) must be applied systematically. With substance abusers, this typically involves some form of biochemical verification of drug abstinence, usually via urinalysis testing. Such testing requires careful planning so that the schedule of testing (frequency) allows optimal detection of substance use and abstinence. Below we describe the testing program used in our studies, which will illustrate the multiple issues involved in and importance of choosing a monitoring schedule that optimizes efficacy. The importance of having a method for objectively and reliably verifying whether a target behavior occurred pertains as well to other target behaviors. Reliance on self-reports of drug use or completion of other therapeutic tasks is not adequate for effective delivery of a CM program.

\section{Contingency Management for Adolescent Substance Abuse}

A number of factors clearly indicate that CM interventions would likely enhance outcomes for adolescent substance abusers. First, the empirically-based principles of behavior and behavior change from which CM strategies are derived are applicable to all humans and 
nonhuman species. Given the clear and robust data supporting CM for adult substance use treatment outcomes, there is strong reason to expect that similar, CM-based intervention alternatives would be effective for adolescents. Second, adolescents rarely seek treatment on their own but instead enter treatment because their parents, school or the judicial system "require" it. Most adolescents show low motivation to quit or remain abstinent and do not typically see their use a significant problem in need of treatment ${ }^{16}$. CM interventions can offer clear incentives (e.g., tangible positive reinforcers) for quitting that are designed to enhance or engender initial or long-term motivation to abstain. Third, these referral agents that direct adolescents into treatment (families, schools, legal authorities) are also potential sources for the implementation of CM strategies that could enhance initial motivation for abstinence and for maintaining treatment gains by continuing to provide $\mathrm{CM}$ following a treatment episode. Such interventions could be effective additions or alternatives to clinicbased treatments.

\section{Adolescent Contingency Managment Research}

Although not clearly labeled as such, CM strategies appear to be at least a small component of the aforementioned, empirically-based family interventions. A number of studies describe use of CM-like procedures as part of the multi-component intervention being examined, e.g., instructing parents to provide rewards and consequences for abstinence or substance use, or other problem behavior targets or instructing therapists to reinforce abstinence ${ }^{3,17-19}$. Unfortunately, the descriptions of these procedures typically do not provide enough detail to determine the type, schedule, magnitude, or monitoring procedures of the CM-type components being administered, and none of these early studies attempted to isolate and test the $\mathrm{CM}$ components. Azrin et al. ${ }^{20}$ did provide such details about their CM program that included structured parental reinforcement of drug-incompatible activities (which could be monitored by parents at home), but did not specifically target drug abstinence per se.

There are a few recent examples of the use of CM to target adolescent tobacco use. Krishnan-Sarin et al. ${ }^{21}$ found that youth participating in a school based tobacco cessation program who received an abstinence-based incentive intervention and CBT had greater rates of tobacco abstinence than youth who received CBT alone. A total of 53\% of CM youth were abstinent for the entire 4 week intervention period, compared to $0 \%$ of the CBT only group. The $\mathrm{CM}$ procedures were reported in a highly replicable fashion and they included twice daily breath $\mathrm{CO}$ assessment in week 1 , fading to every other day in week 4 , an escalating incentive schedule with a reset, payments to both groups for attending sessions, noncontingent payments to the CBT only group for providing samples, earnings of up to $\$ 313.75$ cash in the CM group for 4 weeks of abstinence, and objective and clearly specified definitions of abstinence. In a second trial, Cavallo et al ${ }^{22}$ compared the same CBT + the CM protocol described above to 3 brief (10-15 minute) CBT sessions per week + the same $\mathrm{CM}$ protocol (Frequent Brief Behavioral Intervention/FBBI). They reported a 4 week abstinence rate of $57.1 \%$ in the weekly CBT $+\mathrm{CM}$ condition, not significantly different from the rate of $38.5 \%$ in the $3 \mathrm{x}$ weekly FBBI $+\mathrm{CM}$ condition. A 2-month follow up found that $12.5 \%$ of the weekly CBT+CM youth and $28.6 \%$ of the $\mathrm{FBBI}+\mathrm{CM}$ youth were abstinent, also not significantly different. Adding CM to CBT clearly boosts abstinence rates dramatically in the short term, however, the high relapse rates suggests the need for longer duration interventions.

Another CM program for adolescent smoking, a web-based 30 day intervention, was tested using a reversal design with 4 youth $^{23}$. All four participants achieved abstinence, and three of the four participants met abstinence criteria when incentives were thinned and during a return to baseline phase. The $\mathrm{CM}$ procedures were reported in a highly replicable fashion and they included breath $\mathrm{CO}$ assessment three times per day, an escalating incentive 
schedule with a reset, earnings of up to $\$ 386.75$ cash paid weekly for meeting criteria in all conditions, and objective and clearly specified definitions of abstinence. Of particular note, across the 30 days, $97.2 \%$ of samples were obtained, demonstrating high compliance with the web-based submission of three video clips per day showing the use and results of breath $\mathrm{CO}$ tests. Although larger and longer term studies need to be conducted, this promising method appears feasible and could extend the reach of CM.

There are also a few examples of the use of CM to target adolescent drug use. Henggeler et al. ${ }^{24}$ tested whether an abstinence-based incentive intervention would enhance outcomes in adolescents participating in Drug Court. The CM procedure did not enhance outcomes when added to Drug Court and a comprehensive family based therapy (MST). A possible reason for the lack of a positive CM effect were that all youth (including the comparison group) received incentives and consequences based on urine drug testing results through a 12month Drug Court program. Also, the CM procedures, as detailed in the implementation manual $^{25}$, involved use of some procedures that reflect significant differences from the typical CM procedures used in effective adult studies. Specifically, four weeks of abstinence had to be achieved prior to earning vouchers, earned points were taken away in the form of fines, drug use resulted in a loss of the opportunity to earn vouchers for at least two weeks, and the magnitude of clinic based incentives was fairly low (\$150 maximum in a 12 month period).

Godley et al. ${ }^{26}$ described a CM intervention for adolescent substance users during continuing care provided post-residential treatment. The intervention involved weekly sessions for 12 weeks and used a fishbowl reinforcement program to reinforce participation in personal goal related activities and abstinence, with youth completing $64 \%$ of the activities they specified. To date, they have reported only on the completion of activities by youth in the CM conditions as an index of the feasibility of identifying, verifying, and increasing prosocial, goal-oriented activities among substance using youth.

\section{Development of a Comprehensive Contingency Management-based Treatment}

In an effort to enhance outcomes for adolescent substance abuse (primarily focusing on marijuana), we sought to create a developmentally-appropriate outpatient CM-based intervention 27,28 . This model integrates four empirically-based interventions. First, an abstinence-based reinforcement intervention (voucher program) is utilized to enhance motivation to engage in treatment and engender marijuana and other drug abstinence. Monetary-based, incentives are provided by the clinic for abstinence documented by urine and breath testing. This procedure is highly similar to the oft-replicated, abstinence reinforcement programs effective in adult treatment studies 29,30 .

Second, a parent-directed CM program is employed to further motivate initiation and maintenance of drug abstinence and to better manage other related behavior problems. Parents are likely to consider their adolescent's marijuana use as problematic, and are usually motivated to take action. However, they may not have the skills to effectively change their adolescent's behavior. With careful training and resources, parents (or guardians) are a natural choice for delivering a CM program that could also enhance or engender motivation to abstain. Our parent CM program includes two components. Parents implement a substance monitoring contract (SMC) that specifies positive and negative consequences to be delivered by the parents in response to documented abstinence or use. The Family Management Curriculum (FMC) from the Adolescent Transitions Program (ATP) teaches parents basic principles and skills to decrease problem behaviors and increase prosocial behaviors, and has demonstrated efficacy for treating conduct disorder ${ }^{31}$. Because 
conduct problems often predate and co-occur with adolescent substance abuse ${ }^{32,33}$, we reasoned that targeting conduct problems, in addition to drug abstinence, might further enhance outcomes in treatment for adolescent substance abuse. Parent compliance with family management treatment positively impacts treatment outcome (Nye et al., 1995), hence the third component of this model uses CM to motivate parent participation. Here, parents earn chances to win prizes via an innovative positive reinforcement program ${ }^{34}$ for actively participating in each treatment component.

Fourth, adolescents receive individual therapy (MET/CBT) to enhance motivation and provide coping skills training focused on achieving and maintaining abstinence 35,36 . Weekly 90-minute sessions are held for 14 weeks, with approximately 45 minutes for the individual teen session, and 45 minutes for the parent training session. At the end of the 14 weeks, all families are offered an additional 12 weeks of once weekly substance testing to facilitate parental monitoring and are referred, when appropriate, to other community resources. In summary, the combination of the individual therapy, voucher program, family management curriculum, and incentives for parent participation is designed to increase (a) adolescents' motivation to achieve and maintain abstinence, (b) parents' abilities to use effective parenting to decrease substance use and other behavior problems, and (c) adolescents' coping skills to help them achieve and adapt to a substance-free lifestyle.

\section{Adolescent CM Implementation Details}

In order to facilitate evaluation, generalization, and dissemination of CM approaches to adolescent substance abuse, it is important to provide procedural details about the key implementation variables that influence the efficacy of any CM intervention: schedule, magnitude, type of consequence, target behavior, and monitoring. Our abstinence-based incentive program was designed so that 1) substance use and its absence are readily detected; 2) abstinence is reinforced; 3) substance use results in a loss of reinforcement; and 4) positive reinforcement gleaned from drug abstinence is used to increase non-drug reinforcement. We use the following schedule for our clinic-based CM intervention for marijuana and other substance abstinence. We offer teens the opportunity to earn rewards twice weekly for 14 weeks based on documented substance abstinence. This schedule allows for frequent opportunities for earning reinforcement, while making it highly probable that any marijuana used at any time during a given week would be detected (see below for a detailed discussion of monitoring issues). Regarding magnitude, during Weeks 1-2, participants receive $\$ 5$ vouchers for each specimen provided independent of test results because of the issues summarized below with the prolonged presence of marijuana metabolites in urine. These vouchers reinforce the provision of the urine specimen (attendance and compliance with the program) and serve as priming reinforcers to demonstrate what can be gained via the voucher program. Subsequent to this, during Weeks 3-14 adolescents earn vouchers only if they provide a substance-negative specimen and parents report abstinence (see discussion of target below). The voucher value starts at $\$ 1.50$, escalates by $\$ 1.50$ with each consecutive negative specimen, and a $\$ 10$ bonus is earned for each two consecutive negative results. Vouchers are reset back to their initial value if results were positive, from which they escalate again after three consecutive negative results. This schedule and magnitude of reinforcement is specifically designed to encourage achievement of longer periods of continuous abstinence ${ }^{30}$. The total magnitude earnings for an adolescent who provides 4 valid urine specimens during the first two weeks of treatment and is abstinent throughout Weeks 3-14 is $\$ 590$. Voucher programs with this same schedule and magnitude have been used successfully with adult marijuana users 29,37 .

Monetary-based vouchers are the type of incentive used. Adolescents earned vouchers (coupons reflecting the amount earned that day and the total voucher balance available on that day), each time abstinence is documented as described below. Vouchers earned are 
redeemed for goods or services therapists deemed in concert with the treatment goal of increasing prosocial, non-drug related activities. Examples of voucher purchases include gift cards or certificates to clothing stores, large chain stores, restaurants, and movie theaters; no cash is provided to adolescents.

The target of this CM program is abstinence from marijuana, alcohol and other drugs. Note that in adults, targeting multiple drugs simultaneously is generally a more difficult goal to achieve than targeting the primary abused substance only ${ }^{38}$. Nonetheless, with adolescents we target all substances for the following reasons. First, although marijuana is the primary drug of abuse for most teens enrolled in treatment, they quite frequently use alcohol, occasionally use other drugs such as opiates cocaine, or amphetamines, and of recent commonly report prescription drug misuse. In addition to the obvious potential for harm of these other substances, we felt that parents would not be receptive to a treatment that provides reinforcement for marijuana abstinence in situations where we, or they, detect their teen using other substances typically considered even more harmful than marijuana. Our experience to date using this procedure suggests that placing voucher contingencies on all drugs of abuse with adolescents who primarily abuse marijuana is not problematic. Little other drug use is usually observed, attrition is low, and the majority of adolescents earn incentives for drug abstinence during the intervention.

To monitor the target behavior (substance abstinence) we use the following procedures. Because the primary target substance is marijuana, and in order to develop an appropriate monitoring schedule to detect marijuana abstinence, we considered the following information in developing our schedule and monitoring procedure. Urinalysis testing provides the usual and typically best method for obtaining the documented evidence needed to effectively administer a CM program targeting drug abstinence. With marijuana, such testing poses some unique issues. First, regular, heavy marijuana users are likely to test positive for marijuana use for 1-3 weeks after cessation at detection levels of $50 \mathrm{ng} / \mathrm{ml}$ of 11 nor-9-carboxy-9-tetrahydrocannabinol (THCCOOH), the primary marijuana metabolite, which is an accepted cutoff level for documenting recent abstinence. Thus, in our voucher program, we provide a two-week notice prior to initiating the voucher program that informs clients that it will take two weeks of abstinence from marijuana for them to achieve a negative urinalysis result. Thus, reinforcement for abstinence must be delayed which is the reason for reinforcing participation only during Weeks 1-2. Note that some clinical agencies and researchers have begun to use quantitative or semi-quantitative testing for $\mathrm{THCCOOH}$ levels as a means to differentiate abstinence from residual THCCOOH in the urine. These methods could potentially reduce the need for delaying reinforcement. However, the accuracy of these procedures for differentiating recent abstinence from reduced use or past use depends heavily on very frequent testing in the early weeks of abstinence (daily or almost daily testing is required to reliably interpret the findings), which would be much costlier than the qualitative tests that rely on standardized cutoffs, and pose additional burden for participants.

A second related concern much less frequently encountered is the possibility that a participant can provide a urine specimen that is negative for cannabis use on one day and then positive for cannabis the next day during the early weeks of abstinence. This can occur because marijuana metabolites are stored in the fatty cells and the rate of their release can vary depending on activities like exercise. We have observed this only a handful of times, but it is worth noting.

A third concern is that urinary THCCOOH levels are affected by the dilution factor of the specific urine specimen provided. Many clients either intentionally or inadvertently drink large quantities of liquid that dilute a urine specimen such that a false negative test result 
might be obtained. If possible, a method to screen for dilute specimens should be used as part of the urine toxicology program. Measurement of creatinine is one method to approximate the dilution factor. Invalid specimens (creatinine below $30 \mathrm{mg} / \mathrm{dl}$ ) results in a request to provide a replacement specimen within 4-24 hours. Failure to submit a scheduled specimen or replacement specimen is treated as a positive result, unless the parent provides an appropriate reason for not being able to attend the clinic. Notwithstanding these issues, the methods to detect recent marijuana use described here have been used effectively and without substantial problems in multiple clinical trials. A comprehensive understanding of the urine toxicology process, however, is necessary to facilitate implementation of an effective program.

In order to accurately monitor substance abstinence, we conduct urine tests on a twice per week schedule. This schedule has been used with marijuana using adults, but differs from the original voucher program for cocaine. Our decision to modify the schedule was twofold. First, we felt it was more practical for participants to make two rather than three visits to the clinic per week. Second, we were concerned that a single instance of cannabis use would carryover to multiple urine tests with the more frequent schedule. The twice a week schedule is sufficient to detect most any cannabis use without exacerbating the potential for carryover positive tests. In summary, we believed that the practical advantages and reduction in the carryover problem gained with the twice per week schedule outweighed the potential benefits that might come from using a voucher delivery schedule that would reinforce abstinence more frequently.

An alcohol breath test is performed at each visit, and parents are provided with and trained to use disposable breathalyzers to test for alcohol use at home. All parents are given five disposable breathalyzers that detect breath alcohol levels at .02/bal and above each week to use at home to detect alcohol use. Parents in the CM condition were instructed to ask their youth to take the breath test when parents suspected alcohol use, using the following procedure. Parents asked the teen if he or she used alcohol that day. If the answer was yes, parents did not administer a breath test, and followed steps outlined below for a positive test. If the teen said no, they did not use alcohol, the parent asked him or her to take the breath test. If the breath test was positive or the test was refused, parents implemented the agreed upon consequence (procedure described below) and reported the positive test to the clinic.

Urine specimens are obtained under same-gender, staff observation to minimize risk of provision of invalid or tampered specimens. Specimens are immediately tested onsite for marijuana, cocaine, opioids, benzodiazepines, amphetamines, and methamphetamines using the Enzyme-Multiplied Immunoassay Technique (EMIT). Rapid or quick tests that can be easily obtained and used without extensive training are good alternatives to use of EMIT or other types of analyzers. Note that if either the adolescent or parents report substance use, or a positive urine specimen or breath test is obtained, the adolescent is considered positive for the purpose of CM implementation.

\section{Interventions Implemented by Parents}

In addition to the $\mathrm{CM}$ procedures implemented by clinic staff, we provide explicit instruction to parents in how to use CM procedures at home. First, with guidance from the therapist, parents develop a substance monitoring contract (SMC) that focuses on substance use or abstinence. The contract specifies positive and negative consequences to be delivered by the parents in response to documented abstinence or use (based on results of the aforementioned substance monitoring procedures) (see Figure 2). The consequences are determined via a collaborative process between therapist, parent and adolescent, and revaluated each week during weekly counseling sessions. This contract uses the same target (abstinence), schedule (twice per week), and monitoring method (urine drug testing, breath 
alcohol testing and self/parent reports) as our clinic based CM. Parents individualize the type of consequence (monetary, voucher type system, privileges) and the magnitude of the consequences, and these factors change throughout treatment in response to treatment success or failure.

Parents also receive a comprehensive behavioral parent training program delivered during weekly sessions. The Family Management Curriculum of the Adolescent Transitions program, is used to teach parents basic principles and skills designed to decrease problem behaviors and increase prosocial behaviors ${ }^{39}$. This program, designed to target youth conduct problems seemed likely to yield broad benefits because conduct problems are highly co-morbid with and strong predictors of poor outcomes among treated adolescent substance abusers 40,41 . This curriculum is highly consistent with the CM model, as parents are taught to select and define problem (target) behaviors and track (monitor) those behaviors over time. Parents clearly specify in advance the type of consequences they will use to increase positive behaviors, similar to those used in the substance monitoring contract, the schedule on which consequences will be provided (e.g., daily), and the magnitude of the consequence. Similar procedures are followed to identify consequences for negative behaviors.

We also utilize the Fishbowl method ${ }^{34}$ to enhance parent participation and compliance. Each week, parents are asked to complete six tasks: attend therapy, attend mid-week urine testing appointments, implement the SMC (twice per week), complete homework, and administer breathalyzers. Parents earn one draw from the Fishbowl for each task. Each draw results in obtaining a winning ( $75 \%$ chance) or non-winning slip ( $25 \%$ chance). Winning slips range in value from small (\$1-\$2: 68\% chance), to medium (\$20: 7\% chance), to large prizes (\$100: 1\% chance). Prizes are delivered immediately and included gift certificates to restaurants, ice cream shops, movie theaters, and grocery stores.

\section{Initial Adolescent CM Trial Results}

We completed an initial two-group randomized trial comparing cognitive behavioral therapy (CBT)+CM (abstinence-based reinforcement and parent-based CM) to CBT+parent drug education (PDE; an attention control condition) ${ }^{28}$. Of the 69 youth enrolled (14-18 years of age), 31 met DSM-IV criteria for marijuana abuse, 30 for marijuana dependence, and 8 adolescents did not meet criteria for abuse or dependence, but reported regular marijuana use. As hypothesized, $\mathrm{CM}$ enhanced continuous abstinence outcomes, engendering more weeks of continuous marijuana abstinence during treatment (7.6 wks vs. CBT+PDE 5.1 wks; $\mathrm{p}=.04, \mathrm{~d}=.48$, medium effect). Those in the $\mathrm{CM}$ group were also more likely to achieve $\geq 8$ weeks of continuous abstinence (53\% vs. $30 \%, \mathrm{p}=.06)$ and $\geq 10$ weeks of continuous abstinence (50\% vs. $19 \%, \mathrm{p}=.006)$.

Despite during treatment differences in abstinence, we did not observe a significant between-group difference in abstinence post treatment. There was an increase in marijuana use from discharge to the 9 month follow up, that, while not returning to intake levels, is of significant concern. Across psychopathology and parenting measures, the CM condition tended to show better outcomes, with significant main effects of treatment condition on negative discipline and externalizing. However, the treatment $x$ time effects were not significant on any scale, indicating that adolescents in both conditions improved on measures of internalizing and externalizing psychopathology, and parents in both conditions showed parenting improvements. There was some evidence of better parental monitoring in the $\mathrm{CM}$ condition. Although both groups were given free breathalyzers by their clinicians and instructed in their use, CM mothers administered significantly more breathalyzers during treatment than $\mathrm{CBT}+\mathrm{PDE}$ mothers (mean=12.9 for $\mathrm{CBT}+\mathrm{CM}$ vs. 2.97 for CBT $+\mathrm{PDE}, \mathrm{t}(67)=3.66, \mathrm{p}<.001)$. We hypothesize that $\mathrm{CBT}+\mathrm{CM}$ mothers administered more 
breathalyzers because they were instructed in contingency contracting, providing them with a clear plan to use the testing results.

The lack of significant treatment condition differences during post-treatment were unexpected and may have resulted from low power to detect differences, a more potent effect of the comparison treatment than expected (rates of abstinence appear good in both conditions compared to prior treatment studies), or simply a less potent intervention effect than expected. Particularly relevant to this discussion of contingency management, the comparison intervention included incentives for participation in counseling and twice weekly urine testing; systematically provided test results to parents; and provided weekly counseling and case management to parents. Although this condition was meant to serve as an "attention control" for the CM intervention, we expect that it was active and enhanced outcomes that would have been achieved with MET/CBT alone and might be considered an alternative model warranting future study. In particular, parents in the comparison condition may have responded to the urine drug testing results in a similar manner to parents in the $\mathrm{CM}$ condition (i.e., withdrawal of privileges or other punishment delivered contingently following positive drug tests, rewards delivered contingently following negative drug tests). The likely tendency for at least some parents to respond in this way may have served to make outcomes more similar across conditions. Moreover, the CM incentive program for attendance and participation may have positively impacted retention thereby facilitating the impact of the individual counseling and the parent program.

\section{Real World Application and Challenges}

Despite only a handful of studies demonstrating the potential of CM for adolescent substance abuse, recent reports indicate that clinicians and provider systems have begun to find innovative ways to implement CM into community treatment settings. Lott and Jennicus ${ }^{42}$ describe how they developed a very low cost CM program to enhance attendance, participation, and abstinence in their community-based, intensive outpatient day treatment program. Reasoning that adolescents may be more sensitive to small, low-cost behavioral incentives than adults, they administered a fishbowl reinforcement program that cost less than $\$ 0.50$ per day. While acknowledging that this was not an experimentally rigorous study, the authors point out that in addition to the positive impact of CM on outcomes, the clinical billings generated by increased retention easily exceeded the costs of implementing the CM program.

Henggeler and colleagues ${ }^{43}$ have taken CM dissemination a step further. They trained 432 community therapists who attended a one-day workshop to implement a CM program for adolescent substance abuse. Systematic follow up interviewing showed that $58 \%$ of the therapists who treated a substance-abusing adolescent used CM with at least one youth. They reported little observable or reported resistance to CM adoption in contrast with the common belief that practitioners are resistant to using these methods.

Before discussing potential barriers to dissemination, we offer a few caveats to consider when developing a CM intervention for general implementation. Like other forms of behavioral treatment, it is important to ensure delivery of an effective intervention. To do so with $\mathrm{CM}$ requires adequate knowledge of behavioral principles and the clinical aspects of substance use testing and treatment to design a program with a high likelihood of success. That is, adequate attention needs to be paid to the schedule and magnitude of the reinforcement plan and to the selection of a target that is clinically appropriate and that can be objectively and accurately monitored. Implementation of a weak or poorly designed plan may result in failure and will provide an inadequate test of CM in general. The design and complexity of the plan will also influence the ease with which it can be disseminated. 
Ideally, one must carefully balance these factors along with consideration of cost constraints when developing a CM program. The paucity of research on CM interventions with adolescents and on the parametrics of CM interventions in general poses challenges for selection of a CM model to apply in diverse settings. Providers must carefully extrapolate from existing studies and rely on a combination of clinical experience and expertise in behavioral principles when developing and implementing a CM program that will be effective in a specific treatment setting. A NIDA and SAMHSA Blending Initiative, Promoting Awareness of Motivational Incentives, reflects one of a growing number of excellent publicly available resources to guide the development of CM programs ${ }^{44}$.

The most probable and oft-discussed barrier to implementation of CM interventions is cost. Unfortunately, because most effective CM interventions involve tangible incentives with set costs, $\mathrm{CM}$ programming tends to be viewed differently than costs associated with more traditional types of clinical interventions (psychosocial therapies or medications). A common response to presentations demonstrating the efficacy of $\mathrm{CM}$ is that the treatment system cannot afford incentives or the urine toxicology testing. As mentioned above, there may be many options for development of very low cost CM programs that can add to the efficacy of existing programs. However, more importantly, if CM interventions can significantly enhance outcomes and are the treatment of choice for achieving optimal abstinence rates, it would seem incumbent upon providers to re-valuate the logistics of their clinical operations and budgets and work with the treatment reimbursement systems to develop plans to offer such potent options to their clients.

\section{Conclusion and Future Directions}

The addition of CM-based interventions to the menu of effective treatment alternatives for substance abuse has generated discussion and excitement in both the research and treatment provider communities. Although the research base for applications in adolescent settings is only beginning to appear, the overwhelming positive evidence base for adult substance abuse treatment gives much reason for high expectations. Future research and clinical innovation must continue to stress the need to find even more potent CM models that delineate necessary and optimal parameters and components for effective implementation. A variety of options are needed for the multitude of clinical settings that must intervene with adolescents. Better understanding of the essential components and effective methods for training community providers in the behavioral principles that underlie CM must guide efforts to disseminate effective CM alternatives.

We now know that CM strategies can be effective for retaining clients in treatment, increasing treatment attendance, and promoting abstinence across multiple types of substance abuse and dependence with various clinical populations. We know that reinforcement schedule parameters such as frequency and magnitude impact the potency of $\mathrm{CM}$ interventions. And, we know that clinicians are willing to learn and implement CM. What is now needed to maximize the effectiveness of CM interventions in the greater community is research to better isolate its active components, accumulation of data on its cost effectiveness, and guidelines for how to best utilize resources to maximize cost-benefit ratios. We further suggest that dissemination efforts must include provider training in the basic principles guiding CM, especially if the provider will need to assist parents in developing and implementing a CM program. With adolescents, areas of future focus should include effective use of parental influence, potential application by systems mandating treatment (schools and judicial), and use of technology such as the internet and cell phones to increase access to and reduce costs of effective interventions such as CM. Despite some promising examples of adolescent $\mathrm{CM}$ interventions, there remain large gaps in our knowledge about how to assist the majority of adolescent substance abusers in achieving and 
maintaining abstinence. Paying close attention to the principles that underlie CM may facilitate the development of more effective interventions.

\section{Acknowledgments}

This work was supported by NIDA Grant DA15186, NIAAA Grant AA016917, and the Arkansas Biosciences Institute, the major research component of the Arkansas Master Tobacco Settlement.

\section{References}

1. Waldron HB, Turner CW. Evidence-based psychosocial treatments for adolescent substance abuse. J Clin Child Adolesc Psychol Jan;2008 37(1):238-261. [PubMed: 18444060]

2. Dennis M, Godley SH, Diamond G, et al. The cannabis youth treatment (CYT) study: Main findings from two randomized trials. J Subst Abuse Treat 2004;27:197-213. [PubMed: 15501373]

3. Waldron HB, Slesnick N, Brody JL, et al. Treatment outcomes for adolescent substance abuse at 4and 7- month assessments. J Consult Clin Psychol 2001;69:802-813. [PubMed: 11680557]

4. Compton WM, Pringle B. Services research on adolescent drug treatment. Commentary on "The cannabis youth treatment (CYT) study: Main findings from two randomized trials". J Subst Abuse Treat 2004;27:195-196. [PubMed: 15501372]

5. Higgins ST, Heil SH, Lussier JP. Clinical implications of reinforcement as a determinant of substance use disorders. Annu Rev Psychol 2004;55:431-461. [PubMed: 14744222]

6. Petry NM, Simic F. Recent advances in the dissemination of contingency management techniques: Clinical and research perspectives. J Subst Abuse Treat 2002;23:81-86. [PubMed: 12220605]

7. Stitzer M. Contingency management and the addictions. Addiction Nov;2006 101(11):1536-1537. [PubMed: 17034430]

8. Higgins, ST.; Silverman, K.; Heil, SH. Contingency management in substance abuse treatment. New York, NY: The Guilford Press; 2008.

9. Sulzer-Azaroff, B.; Meyer, GR. Behavior analysis for lasting change. Fort Worth, TX: Holt Rinehart and Winston; 1991.

10. Petry NM, Peirce JM, Stitzer ML, et al. Effect of prize-based incentives on outcomes in stimulant abusers in outpatient psychosocial treatment programs: A national drug abuse treatment clinical trials network study. Arch Gen Psychiatry 2005;62:1148-1156. [PubMed: 16203960]

11. Lussier JP, Heil SH, Mongeon JA, et al. A meta-analysis of voucher-based reinforcement therapy for substance use disorders. Addiction Feb;2006 101(2):192-203. [PubMed: 16445548]

12. Petry NM, Martin B. Low-cost contingency management for treating cocaine- and opioid-abusing methadone patients. J Consult Clin Psychol Apr;2002 70(2):398-405. [PubMed: 11952198]

13. Sindelar J, Elbel B, Petry NM. What do we get for our money? Cost-effectiveness of adding contingency management. Addiction Feb;2007 102(2):309-316. [PubMed: 17222286]

14. Olmstead TA, Sindelar JL, Easton CJ, et al. The cost-effectiveness of four treatments for marijuana dependence. Addiction Sep;2007 102(9):1443-1453. [PubMed: 17645430]

15. Iguchi MY, Lamb RJ, Belding MA, et al. Contingent reinforcement of group participation versus abstinence in a methadone maintenance program. Exp Clin Psychopharmacol 1996;4:315-321.

16. Breda $C$, Heflinger CA. Predicting incentives to change among adolescents with substance abuse disorder. Am J Drug Alcohol Abuse May;2004 30(2):251-267. [PubMed: 15230075]

17. Liddle HA, Dakof GA, Parker K, et al. Multidimensional family therapy for adolescent drug abuse: Results of a randomized clinical trial. Am J Drug Alcohol Abuse 2001;27(4):651-688. [PubMed: 11727882]

18. Henggeler SW, Clingempeel WG, Brondino MJ, et al. Four-year follow-up of multisystemic therapy with substance-abusing and substance-dependent juvenile offenders. J Am Acad Child Adolesc Psychiatry 2002;41(7):868-874. [PubMed: 12108813]

19. Azrin NH, Donohue B, Besalel VA, et al. Youth drug abuse treatment: A controlled outcome study. J Child Adolesc Subst Abuse 1994;3(3):1-16.

20. Azrin NH, McMahon PT, Donohue B, et al. Behavior therapy for drug abuse: A controlled treatment outcome study. Behav Res Ther 1994;32(8):857-866. [PubMed: 7993330] 
21. Krishnan-Sarin S, Duhig AM, McKee SA, et al. Contingency management for smoking cessation in adolescent smokers. Exp Clin Psychopharmacol Aug;2006 14(3):306-310. [PubMed: 16893273]

22. Cavallo DA, Cooney JL, Duhig AM, et al. Combining cognitive behavioral therapy with contingency management for smoking cessation in adolescent smokers: a preliminary comparison of two different CBT formats. Am J Addict Nov-Dec;2007 16(6):468-474. [PubMed: 18058412]

23. Reynolds B, Dallery J, Shroff P, et al. A web-based contingency management program with adolescent smokers. J Appl Behav Anal Winter;2008 41(4):597-601. [PubMed: 19192862]

24. Henggeler SW, Halliday-Boykins CA, Cunningham PB, et al. Juvenile drug court: Enhancing outcomes by integrating evidence-based treatments. J Consult Clin Psychol Feb;2006 74(1):42-54. [PubMed: 16551142]

25. Cunningham, PB.; Donohue, B.; Randall, J., et al. Integrating contingency management into multisystemic therapy. Charleston: Family Services Research Center, Medical University of South Carolina; 2002.

26. Godley SH, Godley MD, Wright KL, et al. Contingent reinforcement of personal goal activities for adolescents with substance use disorders during post-residential continuing care. Am J Addict JulAug;2008 17(4):278-286. [PubMed: 18612882]

27. Kamon JL, Budney AJ, Stanger C. A contingency management intervention for adolescent marijuana abuse and conduct problems. J Am Acad Child Adolesc Psychiatry 2005;44(6):513521. [PubMed: 15908833]

28. Stanger C, Budney AJ, Kamon J, et al. A Randomized Trial of Contingency Management for Adolescent Marijuana Abuse and Dependence. Drug Alcohol Depend. in press.

29. Budney AJ, Higgins ST, Radonovich KJ, et al. Adding voucher-based incentives to coping-skills and motivational enhancement improves outcomes during treatment for marijuana dependence. $\mathrm{J}$ Consult Clin Psychol 2000;68:1051-1061. [PubMed: 11142539]

30. Higgins ST, Budney AJ, Bickel WK, et al. Incentives improve outcome in outpatient behavioral treatment of cocaine dependence. Arch Gen Psychiatry 1994;54:568-576. [PubMed: 8031230]

31. Dishion, TJ.; Kavanagh, K. Intervening in Adolescent Problem Behavior: A Family-Centered Approach. New York, NY: Guilford Press; 2003.

32. Brook JS, Whiteman M, Finch S, et al. Aggression, intrapsychic distress, and drug use: Antecedent and intervening processes. J Am Acad Child Adolesc Psychiatry 1995;34(8):1076-1084. [PubMed: 7665446]

33. Lynskey MT, Fergusson DM. Childhood conduct problems, attention deficit behaviors, and adolescent alcohol, tobacco, and illicit drug use. J Abnorm Child Psychol 1995;23(3):281-302. [PubMed: 7642838]

34. Petry NM, Martin B, Cooney JL, et al. Give them prizes, and they will come: Contingency management for treatment of alcohol dependence. J Consult Clin Psychol 2000;68:250-257. [PubMed: 10780125]

35. Webb, CPM.; Scudder, M.; Kaminer, Y., et al. The Motivational Enhancement Therapy and Cognitive Behavioral Therapy for Adolescent Cannabis Users. Vol. 2. Rockville, MD: Center for Substance Abuse Treatment, Substance Abuse and Mental Health Services Administration; 2001.

36. Sampl, S.; Kadden, R. Motivational Enhancement Therapy and Cognitive Behavioral Therapy for Adolescent Cannabis Users: 5 Sessions. Vol. 1. Rockville, MD: Center for Substance Abuse Treatment, Substance Abuse and Mental Health Services Administration; 2001.

37. Budney AJ, Moore BA, Rocha HL, et al. Clinical trial of abstinence-based vouchers and cognitivebehavioral therapy for cannabis dependence. J Consult Clin Psychol Apr;2006 74(2):307-316. [PubMed: 16649875]

38. Lussier J, Higgins S, Heil S, et al. Voucher-based reinforcement therapy for substance use disorders: A quantitative review. Addiction 2006;101:192-203. [PubMed: 16445548]

39. Dishion, TJ.; Kavanagh, K.; Veltman, M., et al. Family Management Curriculum V2.0: Leader's Guide. Eugene,OR: Child and Family Center Publications; 2003.

40. Randall J, Henggeler SW, Pickrel SG, et al. Psychiatric comorbidity and the 16-month trajectory of substance-abusing and substance-dependent juvenile offenders. J Am Acad Child Adolesc Psychiatry 1999;38:1118-1124. [PubMed: 10504810] 
41. Young SE, Corley RP, Stallings MC, et al. Substance use, abuse and dependence in adolescents: Prevalence, symptoms profiles and correlates. Drug Alcohol Depend 2002;68(3):309-322. [PubMed: 12393225]

42. Lott DC, Jencius S. Effectiveness of very low-cost contingency management in a community adolescent treatment program. Drug Alcohol Depend Jun 1;2009 102(1-3):162-165. [PubMed: 19250774]

43. Henggeler SW, Chapman JE, Rowland MD, et al. Statewide adoption and initial implementation of contingency management for substance-abusing adolescents. J Consult Clin Psychol Aug;2008 76(4):556-567. [PubMed: 18665685]

44. Hamilton, J.; Kellogg, S.; Killeen, T., et al. Promoting Awareness of Motivational Incentives (PAMI). [September 29, 2009].

http://pami.nattc.org/explore/priorityareas/science/blendinginitiative/pami/ 


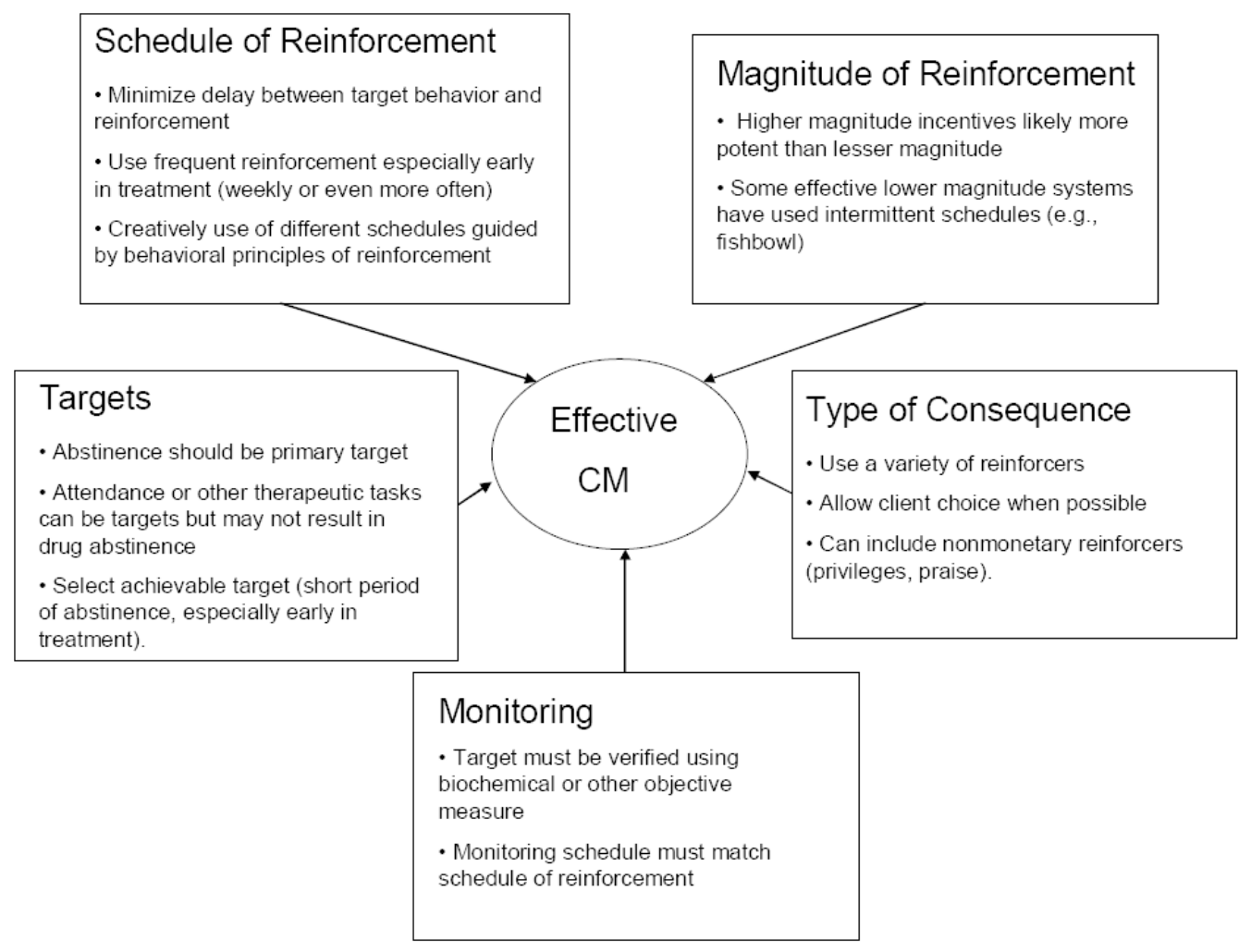

Figure 1.

Key principles in Contingency Management implementation 


\section{Substance Monitoring Contract}

If 's urine drug screen is negative (no drugs detected or reported) and there were no positive or refused alcohol breath tests since the last drug screen, I will:

1. Praise their progress!

2. Ask how I can help them keep up the good work.

3. Celebrate their progress by:

If 's urine drug screen is positive (drugs detected or reported) and/or there were positive or refused alcohol breath tests since the last drug screen, and/or urine screen is refused, I will:

1. Remain calm!

2. $\quad$ Not give a lecture

3. Ask how I can help them

4. Express confidence that they can do better next time

5. Use the following consequence:

\begin{tabular}{lc}
\hline Parent Signature & Date
\end{tabular}

Teen Signature

Date

Therapist Signature

Date

Figure 2.

Substance Monitoring Contract 